\title{
Carpal Tunnel Release through Mini Transverse Approach CTRMTA (Sayed Issa's Approach)
}

\begin{abstract}
Introduction: The idea of CTRMTA technique is enforcement carpal tunnel release through endoscopic carpal tunnel release incision; not using endoscopic instruments but conventional surgical instruments.
\end{abstract}

Methods: Clinical experience with this technique consists of 43 cases and 48 hands over a period of three years, five cases were bilateral release in the same session, this study were from February 2013 to March 2016. All cases were done as outpatient and under local anesthesia.

Results: The mean duration of the operation was 10 minutes, and the minimum duration was 8 minutes. There was no major nerve or vascular injury in all cases. This technique is simple, safe, and highly cosmetically satisfactory for all patients who previously experienced the open standard palmar incision in the other hand, and it is cost effective. Two older than 65 years old female patients suffered pain during first six weeks after surgery, and one of them stopped visiting us after six weeks of surgery, she was not happy because of wrist pain and tenderness after surgery.

Conclusion: It can be used by experienced hand surgeons, especially in countries where endoscopic release is expensive and not available. This technique is simple, safe, cosmetically satisfactory and cost effective.

Keywords: carpal tunnel syndrome, carpal tunnel surgery, carpal tunnel release, ctr, mini-open release, mini incision release, carpal tunnel release through mini transverse approach, ctrmta, endoscopic carpal tunnel release, ectr, open carpal tunnel release, octr, safety, cosmetic, rehabilitation, complications, syria, war, surgery, satisfaction
Volume 10 Issue 3 - 2018

Sayed Issa Abdulhamid

Professor, Adults Nursing Department and Traumatic and Orthopaedics Nursing Faculty, School of Nursing and Midwifery of Aleppo, Syria

Correspondence: Sayed Issa Abdulhamid, Dr. Abdulhamid Sayed Issa's clinic, Al Nile Street, Aleppo, Syria, Tel 00963944838097, 00963-99380639I, Email orthoasi@yahoo.com

Received: March 16, 2018| Published: May 16, 2018

\section{Introduction}

CTRMTA (Sayed Issa's Approach) ${ }^{1}$ can be used by experienced hand surgeons especially in countries where endoscopic release is expensive and not available, as in our country Syria with a set of war circumstances.

CTRMTA is a mini transverse approach technique for carpal tunnel release; the approach is about $1.5 \mathrm{~cm}$ (Figure 1) through distal palmar wrist skin crease incision.

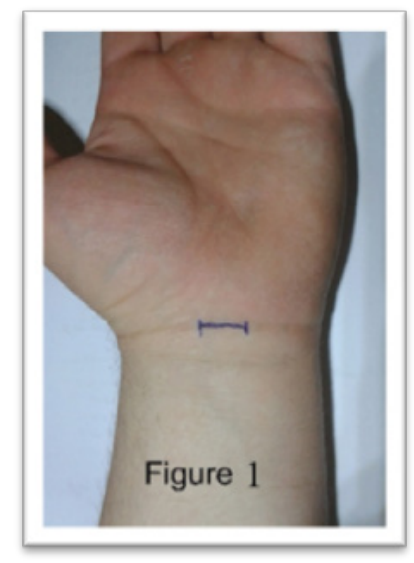

Figure I CTRMTA is about $1.5 \mathrm{~cm}$ through distal palmar wrist skin crease incision.

CTRMTA acquires good rehabilitation achievements on a shorter time, more rapid return to work and daily activities, short healing period, less surgical traumatic incision, less tissues dissection, and less scar tenderness, than in open carpal tunnel release OCTR, or in endoscopic carpal tunnel release ECTR, it causes gentle scar, and very good cosmetic skin healing, the procedure takes about 10 minutes.

The disadvantages of endoscopic carpal release includes: costly equipment required, costly surgery, prolonged procedure time (30-45minutes). Most importantly, there have been many reported complications in association with the procedure, including neuropraxia, transection of the median nerve or its branches, ulnar nerve injury, superficial palmar arch injury. Incomplete division of the transverse carpal ligament has been noted in cadaveric studies and clinical series. ${ }^{2}$

\section{The surgical tools of CTRMTA}
A. One scalpel with blade No. 15
B. Two Senn retractors
C. One curved hemostats
D. One straight Metzenbaum scissors
E. One needle holder
F. One Adson tissue forceps
G. One straight blunt-blunt scissors for cutting the suture
H. One $3 / 0$ nylon suture 


\section{Surgical technique}

The operations were performed through a transverse incision at the distal crease of the wrist on palmar side (Figure 2); we prefer to do it under local anesthesia, to estimate the abnormal sudden pain during ligament cutting to avoid median nerve Irritation. Without using a tourniquet, it is more comfortable from the patient side, but it takes little longer to proceeds because of the few blood Seepage through the incision. It is useful to use a surgical headlight. The wrist is placed in extension over a rolled towel. A $1.5 \mathrm{~cm}$ transverse incision is performed at the distal crease of the wrist in the middle and ulnar to the palmaris longus tendon. The palmaris longus is isolated and retracted radially by putting two Senn retractors; one proximally and the other distally at the ulnar side of palmaris longus, and to retract the skin incision proximally and distally, the antebrachial fascia lying deep to palmaris longus is exposed where it is continuous with the proximal edge of the transverse carpal ligament distally. The anatomic plane between palmaris longus and palmar fascia superficially and the antebrachial fascia and the carpal ligament deeply is opened carefully with scalpel, about $3-5 \mathrm{~mm}$ longitudinal incision is made with the scalpel on the proximal third of the carpal ligament; taking care not to damage the median nerve, thus allowing access to the carpal tunnel. The closed curved hemostats is inserted under the ligament; the tip of curved hemostats is up, and advanced proximally to establish an extrabursal path, where the proximal part of the carpal ligament and the distal portion of the antebrachial fascia are, then incised with a straight Metzenbaum scissors under direct vision, now the proximal edge of the carpal ligament is seen end on, thus one is able to visualize superficial and immediately deep to the carpal ligament. The closed curved hemostats (the tip of curved hemostats is up) is inserted under the ligament and advanced distally to establish an extrabursal path. The proximal edge of the transverse carpal ligament is divided under direct vision by opening the hemostats for more safety, and cutting the ligament by straight Metzenbaum scissors, also it can be made without using the hemostats. In addition, by retracting the palmar skin and fascia volarly, thus providing improved visibility of the distal portion of the carpal ligament, the distal part is transected with the straight Metzenbaum scissors. The transection proceeds snip by snip, at this time ask the patient to flex and extend his fingers to make sure the deeper tip of the scissors are not going inside the deeper tissue, and slips over the flexor sheaths, and carefully with the scissors are directed in the longitudinal axis between the third and the fourth ray. Upon completing the release.

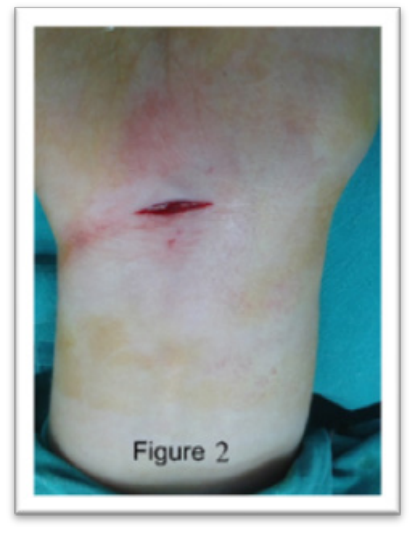

Figure 2 operations were performed through a transverse incision at the distal crease of the wrist on palmar side.
The way to have a safe mini transverse open procedure that we used in the present study is to push the scissors gently towards the longitudinal axis while the tip of the scissors directed volarly and to stop immediately when the ligament resistance suddenly decreases. The incision is also ulnar to the tendon of palmaris longus, which avoids the palmar cutaneous branch of the median nerve. This mini transverse approach is easy to master and have a short learning curve, which contribute to its low morbidity and post-operative complications.

Finally, we check the completeness of the release by introducing the tip of the closed curved hemostats distally, beyond the distal edge of the retinaculum, and withdrawing it slowly while the tip is directed volarly. If the release is incomplete, the surgeon will feel a step; otherwise, withdrawal should be smooth. At this stage, the median nerve will be seen clearly throughout the tunnel.

At the end, the skin incision is closed with one mattress 3-0 nylon suture (Figure 3), and dressed. The patient is advised to start gentle active finger movement immediately, to keep the hand elevated for the first 24 hours, to take a pain killer and NSAID for the next five days and to take an antibiotic for two weeks if necessary for prevention of infection, and to come back after first five days to check the wound, and after two weeks to remove the stitch.

From February 2013 to March 2016; we performed 48 carpal tunnel releases on 43 patients (five cases were bilateral released on the same session), using the CTRMTA. The diagnosis of carpal tunnel syndrome was clinical and then it was confirmed by electrodiagnostic studies in not clear cases. Patients with secondary causes were excluded. All patients had failed prior conservative treatment for at least 3 months before the procedure, except one male patient had a very clear symptoms, including weakness in the hand grip, and a clear first three fingers trembling (thumb, index, and the middle finger).

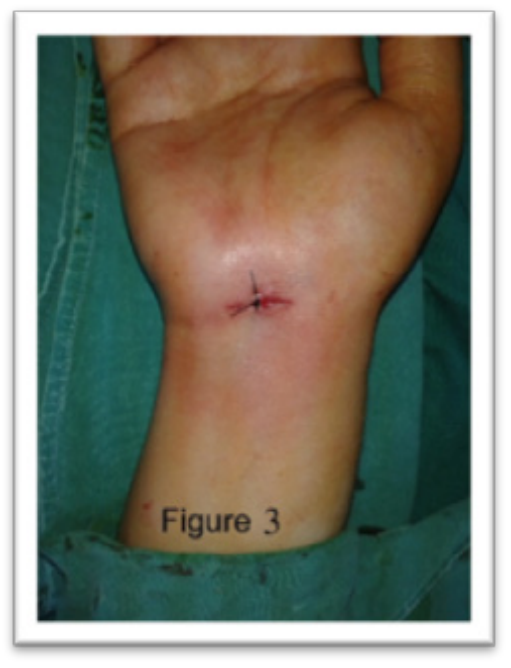

Figure 3 the skin incision is closed with one mattress 3-0 nylon suture, and dressed.

\section{Results}

The mean duration of the operation was 10minutes (8-12minutes). No single major complication, wound or neurovascular, was recorded in any of the patients. Minor complications were observed including: seven superficial wound infections, five wound hematomas resolved 
over the third week without sequel, parasthesia in ulnar nerve distribution in six patients resolved over two months.

Fibrous adhesion effective between subcutaneous tissue from one side and median nerve and tendons sheath in the other side at the skin incision zone, the transverse incision is perpendicularly with the longitudinal axis of carpal tunnel tendons and median nerve; so it is purposive for minimum postoperative fibrosis adhesion.

After a six months follow-up evaluation, all patients were satisfied with their cosmetic results, except one female patient stopped visiting us after six weeks.

Postoperative cosmetic appearance of the palm is an important issue related to skin incision. Our results showed clearly that the CTRMTA we used is good satisfactory and comparable to endoscopic approach. The over all patient satisfaction with the procedure was also excellent at the final follow-up visit after eight months. The longer follow-up period is needed to assess the long term results of this approach regarding the possibility of recurrence of the disease, which was not done in our study.

\section{Discussion}

Various open techniques using small incisions have been described. ${ }^{3-12}$ Carter $^{3}$ published CTR using a transverse wrist incision, with no complications in 100 cases. Bromley ${ }^{9}$ used a short palmar incision, with excellent results. Wilson ${ }^{12}$ used double palmar incisions, with excellent results, using a conventional scissors.

Abouzahr et al. ${ }^{7}$ made CTR in cadavers with a transverse wrist incision, and in one of 28 hands injured the superficial palmar arch, he reported a low complication rate. Hallock et al. ${ }^{8}$ did a comparison of 71 hands which underwent open release using a minimal incision with 66 hands who had ECTR. They found no differences between the two techniques by scar length, complications, and rehabilitation.

Our results show that large improvement in symptoms and function occurred in the first three weeks after surgery, and further improvement continued up to two months postoperatively. These improvements are comparable with those after OCTR and after ECTR. ${ }^{13,14}$

The Average operating time in our series was 10 minutes (range $8-12$ minutes). It is almost similar to the operating times reported by other authors used the same small incision technique. ${ }^{6,11}$ The reduced scar, pillar pain and tenderness of ECTR were better than in the OCTR. ${ }^{14-19}$ In our patients results, were similar to Lee et al. ${ }^{11}$ results.

Reviewing the results of authors who used the mini- open, semiblind approaches revealed major complication rates of $0 \%-0.3 \% .^{3-12}$ Our series showed similar results. The way to have a safe semi-blind open procedure that we used in the present study is to push the scissors gently towards the longitudinal axis between the third and fourth rays while the tip of the scissors directed volarly and to stop immediately when the ligament resistance suddenly decreases. The incision is also ulnar to the tendon of palmaris longus, which avoids the palmar cutaneous branch of the median nerve. These mini-open approaches are easy to master and have a short learning curve, which contribute to their low morbidity and post-operative complications.

Postoperative cosmetic appearance of the palm is an important issue related to skin incision. Our results showed clearly that the CTRMTA we used is highly satisfactory and comparable to endoscopic approach. The over all patient satisfaction with the procedure was also excellent at the final follow-up visit.

\section{Conclusion}

Based on our study and on the results of previous studies, the mini transvers approach technique is simple, easy to master, safe and cost effective. The outcomes which are comparable to endoscopic carpal tunnel release, in many aspects, to open carpal tunnel release justify its use by experienced hand surgeons, ${ }^{20,21}$ especially in countries where endoscopic release is expensive and not widely available.

\section{Acknowledgements}

None.

\section{Conflict of interest}

Authors declare there is no conflict of interest in publishing the article.

\section{References}

1. Sayed Issa A. Carpal Tunnel Release through Mini Transverse Approach (CTRMTA), Sayed Issa's Approach. ClinicalTrials.gov Identifier.

2. Nazerani S, Kalantar Motamedi MH, Nazerani T, et al. Endoscopic carpal tunnel release: a 5-year experience. Trauma Mon. 2014;19(4):e18058.

3. Carter SL. A new instrument a carpal tunnel knife. J Hand Surg Am. 1991;16(1):178-9.

4. Klein RD, Kotsis SV, Chung KC. Open carpal tunnel release using a 1-centimeter incision technique and outcomes for 104 patients. Plast Reconstr Surg. 2003;111(5):1616-22.

5. Cellocco P1, Rossi C, Bizzarri F, et al. Mini-Open Blind Procedure Versus Limited Open Technique for Carpal Tunnel Release: A30-Month Follow-Up Study. J Hand Surg Am. 2005;30(3):493-9.

6. Lee H, Jackson TA. Carpal tunnel release through a limited skin incision under direct visualization using a new instrument, the carposcope. Plast Reconstr Surg. 1996;98(2):313-9; discussion 320.

7. Abouzahr MK, Patsis MC, Chiu DT. Carpal tunnel release using limited direct vision. Plast Reconstr Surg. 1995;95(3):534-8.

8. Hallock GG, Lutz DA. Prospective comparison of minimal incision "open" and two-portal endoscopic carpal tunnel release. Plast Reconstr Surg. 1995;96(4):941-7.

9. Bromley GS. Minimal-incision open carpal tunnel decompression. $J$ Hand Surg Am. 1994;19(1):119-20.

10. Serra JM, Benito JR, MonnerJ. Carpal tunnel release with short incision Plast Reconstr Surg. 1997;99(1):129-35.

11. Lee WB, Strickland JW. Safe carpal tunnel release via a limited palmar incision. Plast Reconstr Surg. 1998;101(2):418-24; discussion 425-6.

12. Wilson KM. Double incision open technique for carpal tunnel release: an alternative to open release. J Hand Surg Am. 1994;19(6):907-12.

13. Okutsu I, Ninomiya S, Takatori Y, et al. Endoscopic management of carpal tunnel syndrome. Arthroscopy. 1989;5(1):11-8.

14. Agee JM, McCarroll HR, Tortosa RD, et al. Endoscopic release of the carpal tunnel: A randomized prospective multicentre study. $J$ Hand Surg Am. 1992;17(6):987-95.

15. Brown RA, Gelberman RH, Seiler JG, et al. Carpal tunnel release: A prospective, randomized assessment of open and endoscopic methods. $J$ Bone Joint Surg Am. 1993;75(9):1265-75. 
16. Agee JM, Peimer CA, Pyrek JD, et al. Endoscopic carpal tunnel release: a prospective study of complications and surgical experience. $J$ Hand Surg Am. 1995;20(2):165-71; discussion 172.

17. Palmer AK, Toivonen DA. Complications of endoscopic and open carpa tunnel release. J Hand Surg Am. 1999;24(3):561-5.

18. Orak MM, Gümüştaş SA, Onay T, et al. Comparison of postoperative pain after open and endoscopic carpal tunnel release: A randomized controlled study. Indian J Orthop. 2016;50(1):65-9.

19. Vasiliadis HS, Nikolakopoulou A, Shrier I, et al. Endoscopic and Open Release Similarly Safe for the Treatment of Carpal Tunnel
Syndrome. A Systematic Review and Meta-Analysis. PLoS One. 2015;10(12):e0143683.

20. Smetana BS, Zhou X, Hurwitz S, et al. Effects of Hand Fellowship Training on Rates of Endoscopic and Open Carpal Tunnel Release. $J$ Hand Surg Am. 2016; 41(4):e53-8.

21. Semple JC, Cargill AO. Carpal tunnel syndrome: Results of surgical decompression. Lancet. 1(7601):918-9. 